\title{
Duas faces do empoderamento: agenciar e promover
}

\author{
Two faces of empowerment: agency and promote \\ Dos facetas del empoderamiento: agenciar y promover
}

Recebido: 11/11/2021 | Revisado: 18/11/2021 | Aceito: 23/11/2021 | Publicado: 05/12/2021

\author{
Manuella Costa da Silva \\ ORCID: https://orcid.org/0000-0002-2702-7452 \\ Universidade de Lisboa, Portugal \\ E-mail: manuellapsi@yahoo.com.br \\ Maria Odília Teixeira \\ ORCID: https://orcid.org/0000-0002-4766-1581 \\ Universidade de Lisboa, Portugal \\ E-mail: moteixeira@psicologia.ulisboa.pt \\ Antonio Pedro de Mello Cruz \\ ORCID: https://orcid.org/0000-0002-6510-2994 \\ Universidade de Brasília, Brasil \\ E-mail: apmcruz@unb.br
}

\begin{abstract}
Resumo
Com enquadramento de modelos ecológicos e empoderamento social, este estudo analisa relações entre contextos e níveis de ansiedade, depressão, controle sociopolítico e perspectiva de futuro dos jovens infratores institucionalizados $(\mathrm{N}=175)$, em três centros do Distrito Federal de Brasília. Numa subamostra $(\mathrm{n}=52)$ é ainda analisada a relação entre dados dos jovens e de profissionais dessas instituições. Na amostra total, a análise de variância (Anova) com fator centro (contexto) em três níveis, os jovens obtêm resultados médios superiores em controle sociopolítico e baixos em ansiedade, num dos centros. Através de tabelas de contingência, observa-se que no mesmo centro, as motivações dos profissionais são mais intensas e há diminuição dos receios de insegurança. Também se observam correlações entre resultados da motivação dos profissionais e os dos jovens em bem-estar e controle sociopolítico. Na conclusão, sublinha-se a tendência sistemática dos dados, em que os níveis de envolvimento sociopolítico e bem estar acompanham a intensidade da motivação intrínseca dos profissionais. Estes dados lançam elementos reflexivos para a intervenção que deve abranger profissionais e adolescentes, tal como preconizado pelas teorias ecológicas e de empoderamento social.
\end{abstract}

Palavras-chave: Ambiente de reabilitação; Jovens infratores; Profissionais socioeducativos; Motivação.

\begin{abstract}
Within the framework of ecological models and social empowerment, this study analyzes relationships between contexts and levels of anxiety, depression, socio-political control and future prospects for young institutionalized offenders $(\mathrm{N}=175)$, in three centers in the Federal District of Brasília. In a subsample $(\mathrm{n}=52)$, the relationship between data from young people and professionals from these institutions is also analyzed. In the total sample, the analysis of variance (ANOVA) with a center factor (context) on three levels, young people obtain higher average results in socio-political control and lower in anxiety, in one of the centers. Through contingency tables, we observed that in the same center, the motivations of the professionals are more intense and there is a decrease in fears of insecurity. In addition, we observed correlations between the results of the motivation of professionals', and the wellbeing and the socio-political control of young people result's. In conclusion, the systematic trend of the data emphasizes that the levels of socio-political involvement and well-being follow the intensity of the professionals' intrinsic motivation. These data provide reflective elements for the intervention that should include professionals and adolescents, as advocated by ecological and social empowerment theories.
\end{abstract}

Keywords: Rehabilitation environment; Young offenders; Socio-educational professionals; Motivation.

\section{Resumen}

En el marco de los modelos ecológicos y el empoderamiento social, este estudio analiza las relaciones entre contextos y niveles de ansiedad, depresión, control sociopolítico y perspectivas de futuro de los jóvenes infractores institucionalizados $(N=175)$, en tres centros del Distrito Federal de Brasilia. En una sub muestra $(n=52)$ también se analiza la relación entre datos de jóvenes e instituciones profesionales. En la muestra total, un análisis de varianza (ANOVA) con un factor central (contexto) en tres niveles, los jóvenes obtienen resultados promedio más altos en control sociopolítico y menores en ansiedad, en uno de los centros. A través de tablas de contingencia, se observa que, en el mismo centro, las motivaciones de los profesionales son más intensas y hay una disminución en los ingresos por inseguridad. También existen correlaciones entre los resultados de la motivación de los profesionales y los jóvenes en el bienestar y el control sociopolítico. En conclusión, se completa la tendencia sistemática de los datos, en la que los 
niveles de implicación sociopolítica y bienestar acompañan a la intensidad de la motivación intrínseca de los profesionales. Estos datos brindan elementos reflexivos para la intervención que debe abarcar a los profesionales y adolescentes, tal y como propugnan las teorías del empoderamiento ecológico y social.

Palabras clave: Ambiente de rehabilitación; Jóvenes ofensores; Profesionales socioeducativos; Motivación.

\section{Introdução}

A Organização Mundial de Saúde perspectiva a violência com complexidades inscritas em padrões de pensamento e comportamento, que são moldados nos contextos familiares, educacionais e comunitários, e em que se perpetuam desigualdades sociais. A criminalidade nos jovens é associada à pobreza e disfuncionalidade familiar, e também aos preconceitos sociais no regresso à vida social (Uceda-Maza \& Alonso, 2017).

O encarceramento dos jovens e a violência são assuntos transdisciplinares que mobilizam esforços e pesquisas em todo o mundo. O problema da violência é agenda da política social, educacional e de saúde mental da maioria das nações, constituindo um dos principais desafios da sociedade (Winter et al., 2017). Na relação entre violência, saúde e educação há a necessidade de identificar fatores do risco, mas sobretudo fatores promotores de agenciamento pessoal que conduzam à mudança, e à eficácia dos programas de prevenção (Krug, 2002).

No Brasil, a internação até 3 anos em centros de detenção juvenil é das medidas socioeducativas mais graves para adolescentes em conflito com a lei. As medidas socioeducativas estão direcionadas para a reabilitação, retorno à vida em sociedade e diminuição da reincidência criminal. A reabilitação, através de ações socioeducativas, privilegia o regresso à vida em sociedade (Uceda-Maza \& Alonso, 2017), ao contrário das medidas dirigidas à culpabilização do infrator. No âmbito legal, o próprio escalonamento das medidas diferencia perfis infracionais. Em meio aberto, há casos em que a intervenção é efetiva e alcança resultados positivos no início da inserção infracional, enquanto em outros casos a reincidência criminal conduz os infratores aos Centros de Internamento, em meio fechado. A literatura aponta uma correlação entre reincidência e aumento da gravidade de delitos (e.g., Peterson \& Robbins, 2008; DeLisi \& Vaughn, 2008; Fine et al., 2018). O levantamento Anual do Sistema Nacional de Atendimento Socioeducativo (SINASE, 2018) identifica 476 unidades de restrição e privação de liberdade. No ano de 2008, o número de jovens em privação de liberdade era de 16.868, e em 2014 aumentou para 24.628; um aumento de $46 \%$. A maioria dos atos infracionais são roubos $(44,41 \%)$, tráfico de drogas $(24,24 \%)$ e 9,47\% para homicídios. Há prevalência de infratores do sexo masculino (95\%), e a maioria dos jovens internados tem entre 16 e 17 anos (56\%). O relatório da Secretaria Nacional de Direitos Humanos também divide os dados segundo a raça, sendo 55,77\% dos jovens pretos ou pardos, $21,16 \%$ brancos, $0,63 \%$ amarelos, $0,25 \%$ indígenas e 22,16\% sem informação. Este relatório demonstra diferenças na gestão governamental das politicas socioeducativas do Brasil, conforme alocação institucional de cada unidade federativa. Em 10 Estados brasileiros, esta responsabilidade cabe à Secretaria de Assistência Social e Cidadania, em 9 à Secretaria de Justiça e Segurança Pública, em 3 à Secretaria do Trabalho, em 2 à Secretaria da Criança e do Adolescente, em 2 à Secretaria de Direitos Humanos e num outro Estado cabe à Secretaria da Educação a execução das medidas.

A Justiça Restaurativa (Zehr, 2008) traz um paradigma moderno de responsabilização, pautado na reparação da vítima e na ressignificação do sujeito. Ao invés de cumprir uma penalidade retributiva focada na punição, procuram-se fatores positivos que tenham impacto no ofensor e também na vítima e na sociedade. As técnicas usadas visam a interação e ressignificação dos atores do processo criminal. Um exemplo é o círculo restaurativo (Ferrão et al., 2016), visto pelas autoras como uma técnica que qualifica a intervenção e traz impactos positivos nas aspirações de futuro dos adolescentes.

O crime traz implicações que afetam esferas políticas e legais, bem como o bem-estar e a necessidade humana de paz e segurança. Segundo Ruffo e Toledo (2017), a perspectiva restaurativa “contribui para o próprio Estado democrático Brasileiro, uma vez que não só restaura o convívio público, desarticulando a violência de direitos, mas também redimensiona a posição das pessoas envolvidas, que passam a ter foco no futuro e no coletivo evitando ainda reações 
sociais desmedidas" (p.131).

\section{Empoderamento Social}

A teoria do empoderamento advém da psicologia comunitária na procura de melhorar a saúde e o bem-estar dos indivíduos, grupos e populações. O empoderamento é um construto multidisciplinar, cuja essência reside na capacitação dos grupos marginalizados para promover justiça e mudança social (Zimmerman \& Eisman, 2017). Empoderamento é um processo pelo qual pessoas, organizações e comunidades obtêm domínio sobre questões que lhes dizem respeito. Empoderamento psicológico inclui engajamento ativo na comunidade e entendimento do ambiente sócio-político, bem como autoperceção de competência social (Zimmerman \& Eisman, 2017). Internacionalmente, Paulo Freire foi um dos primeiros autores a utilizar conceitos de empoderamento a par de conscientização, com a distinção entre empoderamento individual e social, sendo a tónica colocada no segundo conceito na sua procura de transformação social através da educação (Roso \& Romani, 2014). A educação por empoderamento adopta a estratégia participativa dos jovens, identificando seus problemas, planejando e engajamento em ações sociais para mudar condições subjacentes que causam transtornos e doenças.

Chronister e McWhirter (2003, p.422) definem empoderamento como o "processo pelo qual pessoas, organizações ou grupos que são oprimidos ou marginalizados se (a) tornam conscientes da dinâmica de poder no trabalho nas suas vidas, (b) desenvolvem habilidades para ganharem controle razoável sobre suas vidas, (c) o qual eles exercem, (d) sem infringir os direitos de outros, e (e) que coincide com o apoio ativo ao empoderamento de outros em sua comunidade". As questões do empoderamento colocam-se ao nível individual, comunitário e organizacional. O processo de empoderamento inclui reconhecimento e utilização de competências pessoais; consciência do impacto do contexto na vida pessoal; utilização da rede social de apoio; consciência crítica da dinâmica do poder na vida. Essencialmente, o processo de consciência crítica envolve aumentar a consciência do eu (identidade), dos outros (contexto) e da relação do eu com os outros (dinâmicas de poder) e, consequentemente a compreensão do controle em situações da vida. A consciência crítica fortalece a intenção e o comprometimento com a mudança e aumenta a procura pela libertação da opressão.

Uma abordagem de empoderamento envolve necessariamente os jovens no processo de resolver o problema da violência em suas comunidades (Morrel-Samuels et al., 2013). Há uma grande quantidade de pesquisas mostrando que os alunos que foram treinados em processos de autorregulação e estabelecimento de metas exibem altos níveis de motivação e realização (e.g., Cleary \& Zimmerman, 2004). Nesta perspectiva, o desenvolvimento é compreendido sob seus aspectos dinâmicos e a partir da interação entre as influências e interações dessas esferas e influências (Maital e Scher, 2003). Juntos, o empoderamento e a teoria ecológica fornecem uma estrutura conceitual e operacional para programar ações que envolvam os jovens em atividades positivas, preparando-os para se tornarem agentes efetivos de mudança da comunidade (Zimmerman et al. 2011). No Modelo Ecológico de desenvolvimento humano de Bronfenbrenner (1994), o comportamento e a mudança são embutidos na acomodação progressiva entre o indivíduo ativo e os contextos em que está inserido, sendo este processo ligado às relações entre contextos mais próximos (e.g., família, escola) e mais distantes (e.g., politicas sociais e educativas). Assim, as estratégias de análise e intervenção abrangem os níveis individuais, familiares, escolares, sociais, políticos e comunitários. Esta é também uma abordagem positiva da natureza humana (Seligman, 2002), em que é focalizado o florescimento que conduz à saúde, adaptação, mudança e bem-estar. A abordagem de empoderamento enfatiza os fatores com impacto no bem-estar e no desenvolvimento de competência, ao contrário das abordagens que se centram na doença e deficiência. A competência em gerir a própria vida em comunidade é relacionada com autoestima, saúde mental (Teixeira \& Costa, 2017; Zimmerman \& Warschausky, 1998), e capacidade em estabelecer projetos (Lopes \& Teixeira, 2012; Teixeira \& Ferreira, 2018).

A educação e o trabalho são promotores de inclusão social, numa sociedade carregada de medos associados ao crime. No entanto, as pesquisas (e.g., Ebata, Petersen \& Conger, 1990) sobre fatores de proteção sugerem tipos específicos de 
intervenções direcionadas a indivíduos que apresentam fatores de risco específicos. Esses autores salientam a importância da contextualização de mudanças na adolescência em seus aspectos biológicos, cognitivos, relações familiares, de pares sociais e escolares, bem como a influência da autoestima e auto-percepção como moduladores deste processo. As pesquisas no âmbito das teorias de empoderamento social (e.g., Peterson et al., 2011) apontam o significado de desenvolvimento positivo da juventude no âmbito das competências, das opções de vida saudáveis, na confiança para tomar decisões, na conexão afetiva com adultos e pares, na responsabilidade por si e pelos outros, e no sentimento de pertença e participação na comunidade.

Em síntese, a literatura (e.g., Bandura, 1997) aponta o agenciamento como um dos principais componentes de empoderar os jovens para a mudança, sendo este processo inscrito em múltiplas redes de aprendizagem, afetos e confiança, que envolvem o indivíduo, as organizações sociais e educacionais. Este trabalho analisa a relação entre algumas das dimensões psicológicas do agenciamento dos jovens e as características dos ambientes educacionais de reabilitação, nomeadamente o contexto relacional, representado pelos profissionais das instituições.

\section{Métodologia}

\section{Participantes}

A pesquisa incluiu 175 adolescentes homens de três centros, que foram recrutados por conveniência e acessibilidade. Os jovens estavam distribuídos pelos três centros, aqui designados por AAA, BBB e YYY, respetivamente 39\%, 29\% e 33\%. De acordo com o que foi dito anteriormente, a internação surge como última possibilidade de medida socioeducativa aplicável, sendo esta amostra composta por jovens reincidentes e/ou que cometeram crimes de maior gravidade. No Distrito Federal de Brasília existem seis unidades socioeducativas para adolescentes do sexo masculino, que no período deste estudo albergavam 579 jovens (Coordenação de Internação, Subsecretaria do Sistema Socioeducativo, Secretaria de Estado de Justiça e Cidadania do Distrito Federal, comunicação pessoal, 26 de Outubro de 2020). A média de idade dos participantes é de 16.80 (variação 14-20), com média detenção na unidade de internação de $10.73 \pm 7.36$ meses. A média de anos de escolarização formal foi de $8.44 \pm 1.88$ (Tabela 1).

Tabela 1. Características demográficas dos participantes.

\begin{tabular}{ll}
\hline Características da Amostra de Adolescentes & \\
\hline Participantes & $\mathrm{n}=175$ \\
Média ( \pm DP) idade (anos) & $17.00 \pm 1.00$ \\
Média ( \pm DP) anos de escolarização & $8.44 \pm 1.88$ \\
Média ( \pm DP) meses de internação & $11.00 \pm 7.00$ \\
\hline
\end{tabular}

Fonte. Autores (2021).

O estudo também incluiu 52 servidores públicos - agentes socioeducativos (74\%) e especialistas socioeducativos (27\%) (assistentes sociais, psicólogos e pedagogos) que atuavam na unidade AAA (47\%), BBB (20\%) e YYY (33\%). Estes profissionais tinham qualificações superiores, e idades entre 26 e 53 anos $(M=35,44, \mathrm{DP}=6,77)$, sendo $63 \%$ do sexo feminino. O grupo dos 52 jovens é aleatório dentro dos respetivos centros, sendo a distribuição de $49 \%$ no centro AAA, 18\% no BBB e 33\% em YYY. Neste grupo, a média etária é de 16.66 (variação 14-20), e de detenção na unidade de $2.02 \pm 0.90$ meses.

\section{Instrumentos}

\section{Questionário de Dados Pessoais}

Este questionário foi elaborado para a presente pesquisa, e inclui dados demográficos e outros indicadores da história social, emocional, académica e vocacional dos participantes. 


\section{Escala de Sentimentos para o Futuro}

Esta escala inspira-se na literatura (e.g., Moneta, 2014) que descreve os afetos como reações subjetivas de valência positiva ou negativa. Trata-se de uma escala com 10 itens, que avaliam sentimentos positivos (e.g., otimista) e negativos (e.g., angustiado). A resposta tem formato de escala de Likert de 5 pontos (1 - Nada a 5 - Fortíssimo). Os resultados confirmam a independência das duas dimensões, que identificam sentimentos positivos e negativos. Os coeficientes alfa de Cronbach são de 0.80 e 0.65 , para as subescalas sentimentos positivos e negativos, respectivamente (Teixeira et al. 2021).

\section{Escala de Controlo Sociopolítico para a Juventude- SPCS-Y}

A Escala de Controle Sociopolítico para a Juventude (SPCS-Y) (Peterson et al., 2011) é de autorrelato, e avalia a dimensão emocional ou intrapessoal do empoderamento. De acordo com Zimmerman (1995), a dimensão emocional está relacionada com a identificação ao grupo, mas também com autoeficácia individual e coletiva e empoderamento intrapessoal (Speer \& Peterson, 2000). O construto ainda inclui componentes cognitivas e comportamentais (Zimmerman, 1995). O controle sociopolítico é definido como crenças sobre capacidades e eficácia pessoal em sistemas sociais e políticos (Zimmerman et al., 1999).

Na versão original, a SPCS-Y tem 17 itens em forma positiva e divididos nas subescalas liderança (e.g., preferiria ser um líder em vez de um seguidor) e controle de políticas (e.g., eu gosto de participação, porque eu quero ter muito a dizer na minha comunidade ou escola). A resposta é dada em escala de Likert de 5 pontos. Nesta versão portuguesa do Brasil usou-se uma escala de 3 pontos de "sempre", "às vezes" e "nunca", para facilitar a resposta dos participantes. São ainda considerados os resultados para a escala total, pela soma de todos os itens.

Os dados revelam índices favoráveis à consistência interna da medida, sendo os coeficientes alfas de Cronbach de 0,89 para a escala total, de 0,81 em liderança e 0,85 em controle de políticas (Peterson, et al., 2011). Numa amostra portuguesa, os alfas de Cronbach são 0,79 em liderança e .81 em controle de políticas (Rodrigues et al., 2016).

\section{Escala de Desenvolvimento e Bem-Estar}

A Escala de Desenvolvimento e Bem-estar (Diener et al., 2009) é de autorrelato, contém 8 itens cujos conteúdos avaliam relações interpessoais, sentimentos de competência, significado e propósito na vida. As respostas são dadas numa escala de Likert de 7 pontos (1 - Discordo fortemente a 7 - Concordo fortemente). Em diferentes amostras, designadamente portuguesas, há índices de validade e precisão, sobretudo da natureza unifatorial da medida e da consistência interna, com coeficientes alfa de Cronbach superiores a 0.87 (Almeida \& Teixeira, 2018; Diener et al., 2009; Teixeira \& Costa, 2017).

\section{Inventários de Ansiedade (BAI) e Depressão de Beck (BDI)}

O Inventário de Ansiedade é de auto-relato e avalia indicadores de sintomas de ansiedade. Contém 21 itens com resposta em quatro alternativas ( 0 a 3), que correspondem aos níveis de ansiedade. A pontuação total pode variar de 0 a 63 . O Inventário de Depressão é também de auto-relato, com 21 itens e quatro opções de resposta (0-3), que correspondem aos níveis de sintomas de depressão. A pontuação total pode variar de 0 a 63. BAI e BDI mostram alta consistência interna, conforme indicado pelos coeficientes alfa de Cronbach de 0.92 e 0.81, respectivamente (Beck et al., 1988, Gorenstein \& Andrade, 1998). Os pontos de corte do BAI foram 0-7 (ansiedade mínima), 8-15 (ansiedade leve), 16-25 (ansiedade moderada) e 26-63 (ansiedade severa). As pontuações de corte para o BDI foram 0-9 (depressão mínima), 10-16 (depressão leve), 17-29 (depressão moderada) e 30-63 (depressão grave), (Beck et al., 1998; Beck e Steer, 1993; Beck et al., 1996). 


\section{Escala de Percepção do Servidores}

A Escala de Percepção dos Servidores é de autorrelato, com cinco conjuntos de itens que avaliam a motivação no trabalho (e.g., apoiar o outro); mudanças no trabalho (vontade de mudar o contexto de trabalho); eficácia da medida socioeducativa (e.g., avaliação da eficácia da medida socioeducativa); saúde mental (autoavaliação da saúde mental), e dificuldades encontradas no ambiente de trabalho, quer em recursos exteriores (e.g., falta de recursos materiais) quer em recursos intrapessoais (e.g., não acredito no que faço).

\section{Procedimentos}

Éticos

Os jovens participaram de modo voluntário, sendo informados sobre os objetivos da pesquisa e condições do consentimento livre e esclarecido. No caso de maiores de idade o consentimento foi assinado pelos próprios e, no caso dos menores de idade, o assentimento foi assinado pelos responsáveis de cada Unidade, de acordo com as recomendações do Comitê de Ética. A participação dos adolescentes seguiu os seguintes critérios: (1) estar em medida socioeducativa de internação; (2) não interferir nas atividades de rotina institucional; (3) capacidade de leitura, de acordo com o nível mínimo de leitura e compreensão; (4) na mesma sala não incluir jovens com conflitos graves dentro da instituição. A seleção foi realizada por membros da Gerência Psicossocial e de Segurança de cada Unidade.

A autorização legal para coleta de dados foi obtida junto aos órgãos executivos e judiciários do Distrito Federal, sendo as recolhas de dados agendadas com gestores (diretores, gerentes de segurança e gerentes psicossociais), para não interferir nos horários e atividades dos adolescentes. Foi pedida a colaboração dos jovens, sem lhes oferecer qualquer incentivo.

Os pesquisadores tomaram rigorosos cuidados de confidencialidade dos dados, especialmente na identificação dos adolescentes. As informações pessoais foram transformadas em códigos para o tratamento estatístico, e os dados originais deletados. O protocolo foi aprovado no Comitê de Ética Nacional em Pesquisa, Brasil (Protocolo CAEE 34895287), em conformidade com o Código de Ética da Associação Médica Mundial (Declaração de Helsinque).

\section{Recolha dos dados}

Nas diferentes instituições, as observações foram realizadas em grupo (entre 2 e 17 participantes), com a presença do pesquisador (psicólogo com experiência com jovens infratores institucionalizados) e um ou mais membros da equipe de segurança da instituição.

\section{Análise de resultados}

Nos resultados, procedeu-se à análise de variância (Anova) a um fator em três níveis, para identificar o efeito das instituições nos resultados da ansiedade, depressão, agenciamento sociopolítico, sentimentos de futuro e bem-estar. Foram estimadas correlações (Pearson) para analisar a associação entre variáveis motivacionais dos profissionais e dos jovens, no conjunto das instituições. Os dados dos servidores foram ainda submetidos a tabelas de contingência para identificar a relação entre variáveis motivacionais dos profissionais e a variável centro. As análises estatisticas foram realizadas no software SPSS, versão 26.0.

\section{Resultados}

\section{Distribuições e precisão}

A Tabela 2 apresenta indicadores da distribuição dos resultados e consistência das medidas. A amplitude e os desvios padrão indicam variabilidade dos resultados nesta amostra. Nas diferentes medidas, os coeficientes alfa de Cronbach indiciam 
bons níveis de consistência interna

Tabela 2. Estatísticas descritivas e precisão.

\begin{tabular}{l|c|c|c|c|c}
\hline Escalas & Mínimo & Máximo & Média & DP & $\begin{array}{c}\text { Alfa } \\
\text { Cronbach }\end{array}$ \\
\hline Ansiedade & 0 & 50 & 12.78 & 11.06 & 0.91 \\
Depressão & 0 & 52 & 12.36 & 10.42 & 0.90 \\
Bem-estar & 8 & 56 & 38.61 & 11.46 & 0.85 \\
S. Positivos & 5 & 25 & 18.42 & 5.17 & 0.80 \\
S. Negativos & 5 & 21 & 8.54 & 3.59 & 0.70 \\
Liderança & 6 & 35 & 10.94 & 3.14 & 0.71 \\
C. Sócio-político & 10 & 29 & 21.13 & 4.18 & 0.83 \\
Total C. Sócio-político & 16 & 55 & 34.14 & 6.14 & 0.83 \\
\hline
\end{tabular}

Fonte. Autores (2021).

\section{Diferenças de grupos}

$\mathrm{Na}$ análise de variância (Anova) a um fator com três níveis, o efeito centro é significativo nos resultados das escalas Ansiedade $[F(2,151)=6,65 ; p=0,002]$, Controle de Políticas $[F(2,151)=5,88 ; p=0,003]$, e no total do Controle Sócio-político $[F(2,151)=5,62 ; p=0,004]$. O teste de Levene confirma a homogeneidade das variâncias. As comparações múltiplas das médias, por método Scheffé, evidenciam que as médias de ansiedade são significativamente inferiores na instituição YYY comparativamente à instituição AAA $\left(M_{\mathrm{YYY}}=10.13, D P=9.72 ; M_{\text {aaa }}=16.84, D P=11.61 ; p<0.001 ; \eta^{2} \mathrm{p}=0.63\right)$. Os jovens da instituição BBB também revelam médias inferiores aos da instituição AAA $\left(M_{\mathrm{bbb}}=10.58, D P=10.45 ; M_{\text {aaa }}=16.84, D P=\right.$ $\left.11.61 ; p<0.001 ; \eta_{\mathrm{p}}^{2}=0.52\right)$. Nos dois casos, é elevada a dimensão do efeito. Na subescala Controle de Políticas, os resultados médios são superiores na instituição YYY em comparação com a instituição AAA $\left(M_{\mathrm{YYY}}=22.27, D P=4.08 ; M_{\text {aаa }}=19.89\right.$, $\left.D P=4.26 ; p<0.01 ; \eta^{2} \mathrm{p}=0.57\right)$, e a mesma tendência é observada nos resultados Totais da Escala SPCS-Y $\left(M_{\mathrm{YYY}}=35.63, D P\right.$ $=6.42 ; M_{\text {aaa }}=32.29, D P=6.39 ; p<0.01 ; \eta_{\mathrm{p}}^{2}=0.52$ ). Nestes dados, a dimensão do efeito é elevada.

\section{Correlações entre resultados dos jovens e dos profissionais}

Os resultados mostram correlações significativas e positivas entre motivação material (e.g., salário) dos profissionais e bem-estar $(0,35)$ dos adolescentes, bem como entre satisfação e motivação intrínseca (variedade, identificação com contexto, significado, resultados) dos profissionais e agenciamento sociopolítico $(0,37)$ dos jovens. As correlações são ainda positivas entre os resultados das dificuldades de recursos (divergências, falta de recursos materiais e humanos) dos profissionais e o bem-estar $(0,38)$ e sintomas de depressão dos jovens $(-0,34)$, sendo esta último coeficiente negativo. Os resultados dos profissionais que relataram dificuldades relacionadas com os seus sentimentos (exemplo: insalubridade ou descrença) têm relação negativa com os de agenciamento sociopolítico dos adolescentes $(-0,31)$. Há ainda uma correlação significativa e negativa (-.36) entre a vontade de mudança dos profissionais e sentimentos negativos de futuro dos jovens.

\section{Relação entre dados dos servidores e instituição}

No propósito de explorar os dados dos profissionais em cada uma das instituições, procedeu-se à estatística das tabelas de contingência. A relação é significativa entre instituição e os dados das variáveis "significado atribuído às funções" $[\chi 2(8)=16,19, \mathrm{p}<0.01)]$, "vontade de mudar o contexto de trabalho" $[\chi 2(6)=15,56, \mathrm{p}<0.05)] \mathrm{e}$ "sentimento de insegurança" 
[ $\left.\left.\chi^{2}(8)=16,42, p<0.05\right)\right]$. Na análise das frequências obtidas e esperadas, na variável "significado atribuído às funções" os dados sugerem que os profissionais da instituição YYY atribuem mais pontuação às categorias "forte e fortíssimo", enquanto na instituição AAA os casos obtidos são em maior número do que os esperados nas categorias "nada e pouco forte". Na variável insegurança, os profissionais da instituição YYY tem menos casos obtidos do que esperados nas categorias forte e fortíssimo, e os da instituição AAA são mais os obtidos do que esperados. Ainda na variável "vontade de mudar", na instituição YYY as frequências obtidas são mais elevadas do que as esperadas na categoria muito forte, enquanto na instituição AAA as esperadas são menores do que as obtidas.

\section{Discussão}

Numa perspectiva de agenciamento e empoderamento social, este trabalho analisa o efeito dos ambientes educacionais de reabilitação nas dimensões psicológicas de bem-estar, perspectiva de futuro, depressão e ansiedade dos jovens infratores.

Apesar das limitações metodológicas da pesquisa, os dados sugerem que numa mesma instituição os resultados dos jovens e dos profissionais tendem a adquirir diferenciação, comparativamente às outras duas. Na instituição YYY os jovens têm um maior desenvolvimento de controle sociopolítico e menores índices de ansiedade, e nos profissionais há maiores níveis de motivação quer intrínseca quer extrínseca e menores receios de insegurança. Estes indicadores sugerem a natureza holística e sistémica do comportamento no modelo ecológico de Bronfenbrenner (1994), especificamente no âmbito do crime e reabilitação. A estrutura organizacional e os recursos humanos das instituições podem influenciar de maneira significativa a saúde mental, a aprendizagem, os sentimentos de futuro, o bem-estar e o controle sociopolítico dos jovens. De salientar que no âmbito da aprendizagem, a literatura demonstra que níveis elevados de ansiedade comprometem o aprendizado (Brunelle et al., 2020), bem como a médio e longo prazo, influenciam diretamente na sintomatologia depressiva (Alali, 2016; Kalin, 2020).

Nesta pesquisa, nas correlações entre resultados dos jovens e dos profissionais, há indícios de que a motivação dos profissionais está associada positivamente com o bem-estar e agenciamento sociopolítico dos jovens. Os resultados dos profissionais que expressam maiores dificuldades, como por exemplo, falta de recursos físicos e humanos, insegurança, divergências com colegas, ou mesmo mudanças constantes na estrutura gestora, estão ainda associadas positivamente ao bemestar dos adolescentes, e de forma negativa aos índices de depressão. De realçar as correlações negativas entre as dificuldades de natureza pessoal dos profissionais (e.g., descrença) e os resultados do nível de controle sociopolítico dos jovens, e ainda entre a vontade dos profissionais em mudar o contexto de trabalho e a atitude pessimista de futuro dos jovens. Por outro lado, servidores com maior resistência à mudança, também tiveram resultados elevados no sentimento de insegurança dentro dos centros socioeducativos. Estes dados podem significar que profissionais mais empenhados têm maior consciência das dificuldades em recursos materiais, e que isto pode, num mesmo ambiente educativo, estar associado aos fatores que interferem nas condições responsáveis pelo bem-estar dos jovens, enquanto as dificuldades de natureza pessoal dos educadores parecem associar-se às barreiras de desenvolvimento da consciência sociopolítica nos jovens. Estes dados são corroborados pela relação obtida entre engajamento dos profissionais e atitudes dos jovens (García Rubiano et al., 2009). Segundo Maton e Salem (1995), a base para o empoderamento dentro das organizações se relaciona com crenças e objetivos norteadores, autoeficácia e experiência em diferentes funções, suporte social e liderança. Os profissionais motivados e engajados no trabalho de reinserção social de adolescentes infratores, podem ser vistos como estruturas de apoio e engajamento, que propiciem uma visão positiva de futuro, capaz de alterar o destino dos adolescentes. Neste caso, salienta-se que o trabalho profissional do psicólogo em uma unidade de internação, faz interface com diversas especialidades de atuação profissional, de acordo com a Resolução 003 do Conselho Federal de Psicologia (CFP, 2016). 
O conjunto dos dados tendem a confirmar a complexidade do trabalho das unidades de ressocialização (Volpi, 2015), envolvendo aspectos legais, escolares e educativos, saúde, assistência às famílias, bem como outras atividades pedagógicas de esporte, cultura e lazer. Os sentimentos de insegurança impactam negativamente o trabalho dos profissionais e têm consequências para a saúde do trabalhador e para a manutenção da disciplina e organização destes centros. Por outro lado, trabalhos comunitários que priorizam o apoio, a autoeficácia e o empoderamento, são capazes de criar um clima de segurança com consequências positivas para a saúde e para o futuro dos participantes (Cicognani et al., 2020).

No trabalho de Feijó et al. (2017), numa unidade de internação socioeducativa no Rio Grande do Sul, os índices de estresse ocupacional dos trabalhadores são alarmantes. Ambientes de trabalho insalubres, convívio com situações de violência, longas jornadas de trabalho, pressão e constante estado de alerta e dificuldade de convivência entre colegas, foram alguns dos problemas observados e que comprometem a saúde destes trabalhadores.

Este estudo tem limitações relativamente às amostras, em dimensão e representatividade. Apesar da amostra de 175 participantes tenha abordado aproximadamente $1 / 3$ do total de jovens infratores do sexo masculino cumprindo medidas socioeducativas de internação em Brasília, os resultados não podem ser generalizados para o universo de jovens infratores submetidos a medidas socioeducativas semelhantes no Brasil. Também a presença de pelo menos um membro da equipe de segurança durante a aplicação dos instrumentos pode ter inibido os adolescentes em sua livre manifestação de ideias e sentimentos.

Por se tratar de estudo transversal, há limites do recorte temporal que impedem a generalização de dados e reduz a compreensão da evolução do desenvolvimento dos adolescentes. Finalmente, existem as limitações advindas dos questionários de auto relato, bem como das influências das expectativas de desejabilidade social que podem influenciar vieses de resposta características deste tipo de amostra.

\section{Conclusão}

A coerência dos dados mostra a pertinência dos modelos ecológicos e de empoderamento, que abarcam variáveis pessoais, sociais e institucionais e comunitárias. Os dados desta pesquisa constituem ainda nota de reflexão para se equacionar a intervenção a diferentes níveis e com diferentes destinatários. Nos contextos da Justiça Restaurativa, muito do sucesso das intervenções advém da crença positiva dos profissionais relativamente à capacidade de mudança do ser humano agenciador. Assim, nestas instituições com caraterísticas de vulnerabilidade, a intervenção tem necessariamente duas faces para nos jovens agenciar bem-estar e controlo sobre si próprios e o ambiente, e simultaneamente promover confiança e satisfação aos profissionais, que desempenham um papel chave nos processos de ressignificação da vida destes jovens.

Estudos futuros devem aprofundar o entendimento da saúde do trabalhador dentro das unidades de internação, bem como a busca por programas que favoreçam a motivação, identificação com o trabalho e o atendimento das demandas destes profissionais, como segurança e recursos físicos e humanos. Também são recomendáveis novas pesquisas com metodologia de coleta longitudinal para aprofundar e esclarecer a complexa relação entre as variáveis individuais e do ambiente educacional nos esperados processos de mudança. Juntamente com esta perspectiva, deve-se atender à saúde mental e o bem-estar dos jovens atendidos, bem como suas aspirações e projetos. Trabalhadores motivados, com boa saúde mental e com suas demandas ouvidas e encaminhadas, impulsionam um ambiente propício para o agenciamento pessoal e promoção destes jovens em direção a um projeto de futuro condizente com suas capacidades, bem como abre caminhos de apoio para a reinserção social.

\section{Referências}

Alali, T. (2016). The relationship between anxiety, depression and hopelessness among nonclinical sample. European Psychiatry, 33 , S156. https://doi.org/10.1016/j.eurpsy.2016.01.292 
Almeida, B. R., \& Teixeira, M. O. (2018). Bem-estar e adaptabilidade de carreira na adaptação ao ensino superior. Revista Brasileira de Orientação Profissional, 19(1), 19-30. http://dx.doi.org/1026707/1984-7270/2019v19n1p19.

Bandura, A. (1997). Self-efficacy: The exercise of control. New York: Freeman Bao, X. H., \& Lam, S.-f. (2008). Who makes the choice? Rethinking the role of autonomy and relatedness in Chinese children's motivation. Child Development, 79, 269-283. https://doi.org/10.1111/j.1467-8624.2007.01125.x

Beck, A. T., Epstein, N., Brown, G., \& Steer, R. A. (1988). An inventory for measuring clinical anxiety: Psychometric properties. Journal of Consulting and Clinical Psychology, 56(6), 893-897. https://doi.org/10.1037/0022-006X.56.6.893

Beck, A. T., \& Steer, R. A. (1993). Beck Anxiety Inventory Manual. San Antonio, TX: Psychological Corporation.

Beck, A. T., Steer, R. A., \& Brown, G. K. (1996). Manual for the Beck Depression Inventory. San Antonio, TX: Psychological Corporation.

Beck, A. T.; Steer, R. A. \& Garbin, M. G. (1998) - Psychometric Properties of the Beck Depression Inventory: Twenty-Five Years of Evaluation. Clinical Psychology Review 8(1), 77-100. https://doi.org/10.1016/0272-7358(88)90050-5

Brasil (1988). Constituição da Republica Federativa do Brasil de 1988. http://www.planalto.gov.br/ccivil_03/Constituicao/Constituicao.htm

Bronfenbrenner, U. (1994). Ecological models of human development. International Encyclopedia of Education, 3 (2), $37-43$.

Brunelle, K., Abdulle, S., \& Gorey, K. M. (2020). Anxiety and Depression Among Socioeconomically Vulnerable Students with Learning Disabilities: Exploratory Meta-analysis. Child \& Adolescent Social Work Journal, 37(4), 359-367. https://doi.org/10.1007/s10560-019-00631-w

Cleary, T. J. \& Zimmerman, B. J. (2004). Self-regulation Empowerment Program: a school-based program to enhance self-regulated and self-motivated cycles of student learning. Psychology in the Schools, 41(5), 537-550.

Conselho Federal de Psicologia - CFP (2016). Resolução n 003, de 19 de dezembro: Altera a Resolução CFP n.o 013/2007, que institui a Consolidação das Resoluções relativas ao Título Profissional de Especialista em Psicologia e dispõe sobre normas e procedimentos para seu registro. Disponível em: https://site.cfp.org.br/wp-content/uploads/2016/04/Resolução-003-2016.pdf

Chronister, K. M. \& McWhirter, E. H. (2003). Applying Social Cognitive Career Theory to the Empowerment of Battered Women. Journal of Couseling \& Development, $81,418-425$.

Cicognani, E., Albanesi, C., Valletta, L., Prati., G. (2020) Quality of collaboration within health promotion partnerships: Impact on sense of community, empowerment, and perceived projects' outcomes. Journal of Community Psychology, 48, 323-336. https://doi.org/10.1002/jcop.22254

Diener, E., Wirtz, D., Tov, W., Kim-Prieto, C., Choi, D., Oishi, S., \& Biswas-Diener, R. (2009). New measures of wellbeing: Flourishing and positive and negative feelings. Social Indicators Research, 39, 247-266.

Feijó F. R., Kersting I., Bündchen C., \& Oliveira P. A. B. (2017). Occupational stress in workers from a socio-educational assistance foundation: prevalence and associated factors. Revista Brasileira de Medicina do Trabalho, 15(2), 124-133.

Ferrão, I. S., Santos, S. S. \& Dias, A. C. G. (2016). Psicologia e Práticas Restaurativas na Socioeducação: Relato de Experiência. Psicologia: Ciência e Profissão, 36(2), 354-363. https://doi.org/10.1590/1982-3703000122014

Fine, A., Baglivio, M. T., Cauffman, E., Wolff, K. T., \& Piquero, A. R. (2018). Does the Effect of Self-Regulation on Adolescent Recidivism Vary by Youths' Attitudes? Criminal Justice \& Behavior, 45(2), 214.

García Rubiano, M., Gómez Navarro, P., \& Londoño, O. P. (2009). Relación entre motivación y resistencia al cambio en personas que trabajan en una empresa del sector público, en Bogotá (Colombia) / Relationship among motivation and resistence to change in workers of a public sector company in Bogotá (Colombia). Diversitas: Perspectivas En Psicología, 5(1), 141-159.

Gorenstein, C. \& Andrade, L. (1998). Inventário de Depressão de Beck: propriedades psicométricas da versão em português. Revista de Psiquiatria Clínica, $25(5), 245-250$.

Kalin, N. H. (2020). The critical relationship between anxiety and depression. The American Journal of Psychiatry, 177(5), 365-67. https://doi.org/10.1176/appi.ajp.2020.20030305

Krug, E. G. et al., eds. (2002). World report on violence and health. Geneva, World Health Organization.

Lopes, A. R. \& Teixeira, M. O. (2012). Projetos de carreira, autoeficácia e sucesso escolar em ambiente multicultural. Revista Brasileira de Orientação Profissional, 13(1), 7-14.

Maital, S. \& Scher, A. (2003). Consulting about young children: an ecosystemic developmental perspective. In E. Cole \& J. A. Siegel (Eds.). Effective consultation in School Psychology, 336-364. Göttingen: Hogrefe \& Huber.

Maton, K. I., \& Salem, D. A. (1995). Organizational characteristics of empowering community settings: A multiple case study approach. American Journal of Community Psychology, 23(5), 631-656.

Moneta, G. B. (2014). Positive psychology. A critical introduction. London: Palgrave Macmillan.

Morrel-Samuels, S., Zimmerman, M. A. \& Reischl, T. M. (2013). Creating safe and healthy futures. Reclaiming Children and Youth, 22 (3), $31-36$.

Peterson, M. \& Robbins, B. (2008). Using the MMPI-A to predict recidivism in adjudicated minors. Applied Psychology in Criminal Justice, 4 (2), $172-181$.

Peterson, N. A., Peterson, C. H., Agre, L., Christens, B. D., \& Morton, C. M. (2011). Measuring youth empowerment: Validation of a sociopolitical control scale for youth in an urban community context. Journal of Community Psychology, 39(5), 592-605. 10.1002/jcop.20456. 
Rodrigues, S. S, Coimbra, J. L., Correia, A. N., \& Sousa, V. E. (2016). Escala do controlo sociopolítico para Adolescentes: SPCS-Y. Análise Psicológica, 2 (XXXIV): 177-188. 10.14417/ap.1041

Roso, A., \& Romanini, M. (2014). Empoderamento individual, empoderamento comunitário e conscientização: um ensaio teórico. Psicologia e Saber Social, $3(1), 83-95$

Ruffo, M., \& Toledo, C. (2017). A responsabilização do adolescente na justiça restaurativa. Revista Vianna Sapiens, 6(2), 36, $102-137$. https://www.viannasapiens.com.br/revista/article/view/169

Seligman, M. E. P. (2002). Positive Psychology, Positive Prevention, and Positive Therapy. In: Snyder, C. R. \& Lopez S. J. (Eds.) Handbook of positive psychology. P. 3-9. New York: Oxford University Press.

Sistema Nacional de Atendimento Socioeducativo - SINASE (2018). Brasil, Ministério da Mulher, da Família e dos Direitos Humanos, Levantamento Anual. https://www.gov.br/mdh/pt-br/sdh/noticias/2018/janeiro/divulgado-levantamento-anual-do-sistema-nacional-de-atendimento-socioeducativo.

Speer, P.W., \& Peterson, N.A. (2000). Psychometric properties of an empowerment scale: Testing cognitive, emotional, and behavioral domains. Social Work Research, 24(2), 109-118. https://doi.org/10.1093/swr/24.2.109

Teixeira, M. O. \& Costa, C. J. (2017). Carreira e bem-estar subjetivo no ensino superior. Revista Brasileira de Orientação Profissional, 18(1), 19-29.

Teixeira, M. O., \& Ferreira, I. A. (2018). Fatores familiares e escolares nas aspirações, na autoeficácia e no sucesso académico: um estudo com estudantes de $9^{\circ}$ ano. Revista Portuguesa de Psicologia, 47, 44-60.

Teixeira, M. O., Silva, M. C., Laranjeira, M. \& Veríssimo, A. (2021). Sentimentos de futuro e objetivos. Um estudo com jovens infratores institucionalizados. IV Seminário Internacional de Desenvolvimento de Carreira e Aconselhamento - Desenvolvimento e bem-estar nos contextos de trabalho e de formação (online). Universidade do Algarve, Portugal.

Uceda-Maza, F. X., \& Alonso, J. D. (2017). Vinculación entre la vulnerabilidad y la exclusión social y las trayectorias delictivas. Un estudio de asociación. Psychosocial Intervention, 26(1), 29-37. https://doi.org/10.1016/j.psi.2016.07.003

Volpi, M. (2015). O adolescente e o ato infracional (10th ed.). Cortez.

Winter, K., Spengler, S., Bermpohl, F., Singer, T., \& Kanske, P. (2017). Social cognition in aggressive offenders: Impaired empathy, but intact theory of mind. Scientific Reports, 7(1), 1-10. https://doi.org/10.1038/s41598-017-00745-0

Zehr, H. (2008). Trocando as lentes: um novo foco sobre o crime e a justiça. Palas Athena.

Zimmerman, M. A. (1995). Psychological empowerment: Issues and illustrations. American Journal of Community Psychology, 23(5), 581-599. https://doi.org/10.1007/BF02506983

Zimmerman, M. A. \& Eisman, A. B. (2017). Empowering interventions: strategies for addressing health inequities across levels of analysis. In Bond, M. A., Serrano-Gracía, I. e Key, C. B. APA Handbook of Community Psychology: vol. 2. American Psychological Association. Cap. 11.

Zimmerman, M. A., Ramirez-Valles, J., \& Maton, K. I. (1999). Resilience among urban African American male adolescents: A study of the protective effects of sociopolitical control on their mental health. American Journal of Community Psychology, 27(6), 733-751.

Zimmerman, M. A., Stewart, S. E., Morrel-Samuels, S., Franzen, S. \& Reischl, T. M. (2011). Youth Empowerment Solutions for Peaceful Communities: Combining Theory and Practice in a Community-Level Violence Prevention Curriculum. Health Promotion Practice,12 (3), 425-439 $10.1177 / 1524839909357316$

Zimmerman, M. A. \& Warschausky, S. (1998). Empowerment Theory for Rehabilitation Reserch: Conceptual and Methodological Issues. Rehabilitation Psychology, 43(1), 3-16. 